\title{
Lumacaftor/ Ivacaftor improves exercise tolerance in patients with Cystic Fibrosis and severe airflow obstruction
}

\author{
Peter A. B. Wark ${ }^{1,2^{*}}$ D, Kim Cookson², Theeba Thiruchelvam², John Brannan² and Douglas J. Dorahy ${ }^{1}$
}

\begin{abstract}
Background: Treatment of patients with Cystic Fibrosis homozygous for the Phe508del gene, with Lumacaftor /Ivacaftor (LUM/IVA) improves outcomes in patients with FEV1 > 40\% predicted. We set out to observe the most sensitive clinical measure that would change with treatment in terms of exercise capacity or lung function in adults with severe lung disease as defined by an FEV1 $<40 \%$ predicted when clinically stable.

Methods: 10 adults homozygous for the Phe508del received LUM/IVA. We assessed; six minute walk test (6MWT), spirometry, gas transfer (DLCO), plethysmography, and nitrogen multiple breath washout (MBW) at baseline, 4, 12, 24 and 52 weeks. Comparison was made with 10 matched historical controls that had been observed over 12 months.

Results: There was a significant improvement in $6 \mathrm{MWT}$ by 4 weeks of treatment; with a mean increase of $78 \mathrm{~m}$ (SD 62.3) and this increased to $118.1 \mathrm{~m}$ (SD 80.9) (ANOVA $p=0.006$ ) by 52 weeks. Significant improvements were also seen in the resting heart rate and the oxygen saturation ( $\mathrm{SaO} 2)$ after 6 min walking. A significant improvement was not seen in FEV1 though until 24 weeks, though this was maintained at 52 weeks (ANOVA, $p=0.0004$ ). There were no significant differences seen in the MBW or DLCO. After 12 months treatment with LUM/VA, in comparison to historical controls; the 6MWT increased by $118 \mathrm{~m}$ (SD 80.9), but fell in the controls - $61.3 \mathrm{~m}$ (SD 31.1). FEV1; LUM/IVA led to an increase of $0.398 \mathrm{~L} / \mathrm{min}$, compared to a fall in the controls - 0.18 (SD 0.2).

Conclusion: In adults homozygous for Phe508del with severe disease, treatment with LUM/IVA results in a clinically significant improvement in 6MWT that was evident at 4 weeks and maintained at 52 weeks. Improvement in exercise tolerance is an important outcome to consider in those with more severe airways disease.

Trial registration: This was an observational trial conducted on individuals who became eligible to receive LUM/IVA. All investigations were carried out as part of routine clinical care. The trial was registered in retrospect on the 13/5/ 2019 on the Australian New Zealand Clinical Trials registry; ACTRN12619000708156.
\end{abstract}

Keywords: Cystic fibrosis, Lumacaftor, Ivacaftor, Six minute walk test, Spirometry, Lung transplantation

\section{Background}

Cystic Fibrosis (CF) is a genetic disorder caused by mutations in the gene that encodes for the Cystic Fibrosis Transmembrane Regulator (CFTR) protein, an epithelial ion channel that is crucial in regulating the flow of negatively charged ions across membranes and ensuring adequate hydration of mucus as well as influencing

\footnotetext{
* Correspondence: Peter.wark@newscastle.edu.au

${ }^{1}$ Centre for Healthy Lungs, Hunter Medical Research Institute, University of Newcastle, Lookout Rd New, Lambton, NSW 2305, Australia

${ }^{2}$ Adult Cystic Fibrosis Centre, Department of Respiratory and Sleep Medicine, John Hunter Hospital, Newcastle, Australia
}

immune responses in ways that are incompletely understood [1]. As a consequence, dysfunction results in a multisystem disorder, that seriously impacts upon on the lungs leading to recurring airway infections, progressive airway wall damage, bronchiectasis, fibrosis and airflow obstruction, eventually leading to death or the need for lung transplantation. Lumacaftor/Ivacaftor (LUM/IVA) is a combination agent that targets CFTR. In the case of the most common CF mutation, Phe508del, Lumacaftor acts as a corrector of the dysfunctional protein allowing increased surface expression and Ivacaftor a potentiator, increasing function [2]. In patients older than 12 years,

(c) The Author(s). 2019 Open Access This article is distributed under the terms of the Creative Commons Attribution 4.0 International License (http://creativecommons.org/licenses/by/4.0/), which permits unrestricted use, distribution, and 
homozygous for the Phe508del mutation, and with a percent predicted (pp)FEV1 40-80\%, treatment with LUM/IVA for 24 weeks led to a modest $2.6-4 \%$ increase in FEV1, however improvements were also seen in BMI and there was notably a $30-39 \%$ reduction in the pulmonary exacerbation rate [3]. Furthermore, assessment of this group after 96 weeks of treatment in comparison to matched registry controls, demonstrated a sustained impact with a reduction in the annual rate of decline in FEV1 [4].

The ability to extrapolate these findings to a more severe cohort with CF though is not clear. Further analysis of the original study cohort when stratified by lung function demonstrated that those with the most severe airflow obstruction showed a similar improvement in FEV1 compared to study participants with higher lung function [5]. Though it is unclear whether this small change in lung function would deliver a clinically meaningful benefit to subjects with more severe disease $(<40 \%$ ppFEV1). Finally, it is also unclear what clinical measure if any would be sensitive enough to detect a change, in those with advanced lung disease.

Our aim was to determine a sensitive clinical measure of change, in patients with $\mathrm{CF}$ and severe airways disease as measured by an $\mathrm{FEV} 1<40 \%$ when stable, following treatment with LUM/IVA. We assessed response with spirometry and compared this to changes seen in exercise capacity as measured by the six minute walk test (6MWT), the nitrogen multi-breath washout test, the carbon monoxide gas transfer factor and lung volumes measured by plethysmography.

\section{Methods}

We assessed the response in 10 adult participants from the John Hunter Hospital adult CF centre, aged $\geq 18$ years, homozygous for Phe508del mutation, eligible for access to LUM/IVA through a compassionate access programme provided by Vertex. To be eligible they had to have an FEV1 $\leq 40 \%$ when clinically stable, or experienced $\mathrm{a} \geq 20 \%$ fall in FEV1, or had been referred for lung transplantation assessment. Participants received Lumacaftor $400 \mathrm{mg}$ and Ivacaftor $250 \mathrm{mg}$ twice daily for 12 months.

LUM/IVA participants had the following assessments observed after accessing the compassionate access programme; spirometry, nitrogen MBW test and 6MWT performed on the day of commencing treatment, 4 weeks later, 12 weeks, 24 weeks and at 52 weeks. Plethysmography and single breath carbon monoxide gas transfer factor (DLCO) were performed at baseline, 24 weeks and 52 weeks.

These participants were compared to 10 adult historical controls, who attended the same centre from 2012 to 2015 , aged $\geq 18$ years, homozygous for the Phe508del mutation, who also would have been eligible for access to LUM/IVA as above, who either had ppFEV $1 \leq 40 \%$ when clinically stable at their annual review and or were judged to require referral for transplant at this time. Assessments were taken when clinically stable for their annual review, at baseline and again at the annual review approximately 12 months later. This included spirometry, plethysmography, DLCO and 6MWT.

Spirometry [6], DLCO [7], plethysmography [8] and 6MWT [9] were performed according to ATS/ERS standards. The Nitrogen MBW was performed using an EcoMedix Exhalyzer D running Spiroware Version 3.1.6 software. MBW technique adhered to the guidance of the American Thoracic Society (ATS) [8]. Only the LUM/IVA treated patients underwent MBW testing to determine lung clearance index (LCI) 2.5 functional residual capacity (FRC), sCOND (ventilation heterogeneity generated in the conductive lung zone) and sACIN (ventilation heterogeneity generated peripheral to the acinar entrance). The latter indices were analysed to determine if the region where ventilator inhomogeneity might be most impacted by LUM/IVA treatment could be demonstrated. All data for MBW was generated by the EcoMedix software.

Analysis of continuous variables was performed using parametric techniques to compare differences as these were normally distributed. This was done using a oneway ANOVA with Dunnett's multiple comparisons test. Categorical comparisons were assessed using Fisher's exact test. Analysis was carried out using Graphpad Prism version 7 . The study was approved by the Hunter New England LHD Ethics committee. Subjects agreed to participate with written informed consent.

\section{Results}

Participants are described in Table 1. Both groups were matched in terms of age, sex, the presence of diabetes requiring insulin therapy and exacerbations in the previous 12 months requiring treatment with intravenous antibiotics. Both groups also had severe airflow limitation and air trapping. They also demonstrated a mildly impaired 6MWT distance.

In the 10 participants that received treatment with LUM/IVA, there was a significant improvement in exercise tolerance seen by 4 weeks. This was demonstrated by an increased change in $6 \mathrm{MWT}$ distance after 4 weeks of treatment; mean increase $74 \mathrm{~m}$ (SD 67.1) and this progressively increased by 12 months to $118.1 \mathrm{~m}$ (SD 80.9) (ANOVA $p=0.0018$ ) (Fig. 1). A similar response was seen in total 6MWT distance (Fig. 1). There was a progressive decrease in resting or baseline heart rate at the commencement of the 6MWT, there was also an improvement seen in the fall in $\mathrm{SaO} 2$ after 6 min that was seen by 4 weeks and maintained at 52 weeks (Fig. 1). 
Table 1 Subject characteristics

\begin{tabular}{|c|c|c|c|}
\hline & Received LUM/IVA & Not received LUM/IVA & \\
\hline Number & 10 & 10 & \\
\hline Age (range) years & $26.5(19-56)$ & $30.6(24-47)$ & $P=0.9$ \\
\hline Sex M:F & $6: 4$ & $5: 5$ & \\
\hline BMI & $21.5(3.3)$ & $21.8(3.0)$ & $P=0.8$ \\
\hline FEV1\% predicted & $36.3(2.8)$ & $40.1(6.6)$ & $P=0.1$ \\
\hline Range & $(32.4-40.1)$ & $(32.1-44.3)$ & \\
\hline FVC\% predicted & $66.4(12.2)$ & $66.5(12.2)$ & $P=0.8$ \\
\hline Range & $(51.4-77.2)$ & $(50.8-76.5)$ & \\
\hline TLC (\% predicted) & $98.11(16)$ & $95.7(1.4)$ & $P=0.8$ \\
\hline FRC (\% predicted) & $122(36.3)$ & $124(15.1)$ & $P=0.8$ \\
\hline RV (\% predicted) & $200.4(69.6)$ & $198(53)$ & $P=0.7$ \\
\hline DLCO (\% predicted) & $58(14.6)$ & $63(18.2)$ & $P=0.6$ \\
\hline $6 \mathrm{MWT}$ m & $532.4(90.1)$ & $546(81.3)$ & $P=0.8$ \\
\hline CF related diabetes & 5 & 5 & \\
\hline Pseudomonas & 9 & 9 & \\
\hline Burkholderia Cenocepacia & 1 & 1 & \\
\hline Number of exacerbations in the previous 12 months requiring IV antibiotics & $3(4.6)$ & $2.8(5.6)$ & $P=0.8$ \\
\hline Using regular nebulised DNase & 10 & 10 & NA \\
\hline Using regular Azithromycin & 8 & 9 & $P=0.9$ \\
\hline Using regular nebulised antibiotics & 9 & 9 & $P=0.8$ \\
\hline
\end{tabular}

Change in 6MWT distance

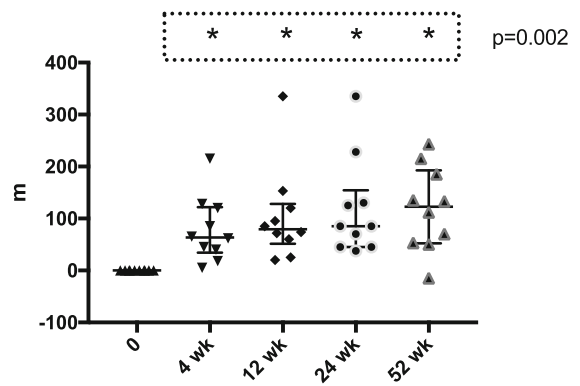

Resting heart rate

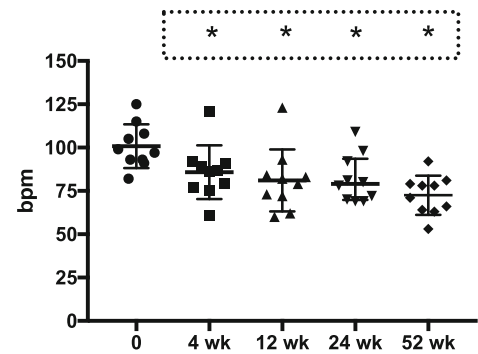

6MW distance

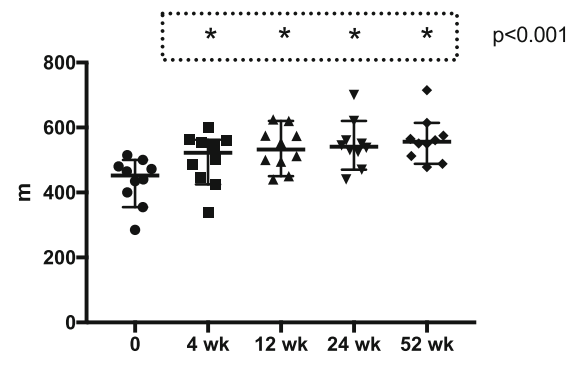

$\mathrm{SaO2}$ at $6 \mathrm{mins}$

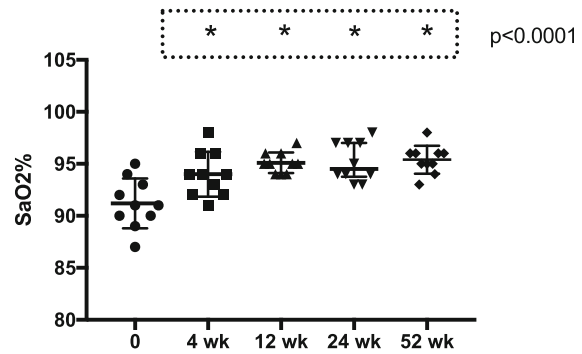

Fig. 1 Data on the 6MWT. The data relates to the 10 subjects treated with LUM/IVA over 12 months. Individual data points are represented as well as the median and interquartile range. Analysis was done using one-way ANOVA with Dunnett's multiple comparisons test. The overall ANOVA $p$ value is given. The groups that are different from baseline $(p<0.05)$ are represented by * 
In comparison, a significant improvement was not seen in FEV1 until after 24 weeks, though this was maintained at 52 weeks (ANOVA, $p=0.0004$ ). This represented a mean increase from baseline in FEV1 by 6 months of $28.8 \%$ (sd 20.1) and by 12 months $23.2 \%$ (SD 18.7) (Fig. 2). At 12 months this represents a relatively small, though significantly different absolute mean increase in FEV1, $0.398 \mathrm{~L} / \mathrm{min}(0.34)$ from baseline $(p<$ 0.001). Similarly, there was an increase in FVC (Fig.1), that became significant after 24 weeks; 26.2\% (SD 16.8) and was maintained at 52 weeks $23.2 \%$ (SD 18.7) (ANOVA $p=0.001$ ). This was a mean absolute increase of $0.492 \mathrm{~L}$ (SD 0.6) after 12 months.

To determine if small changes in lung function could be discerned earlier than 24 weeks the nitrogen MBW was also performed and the lung clearance index (LCI 2.5), FRC, sCOND and sACIN measured at tidal volume breathing (Fig. 3) were derived. The LCI 2.5 was quite

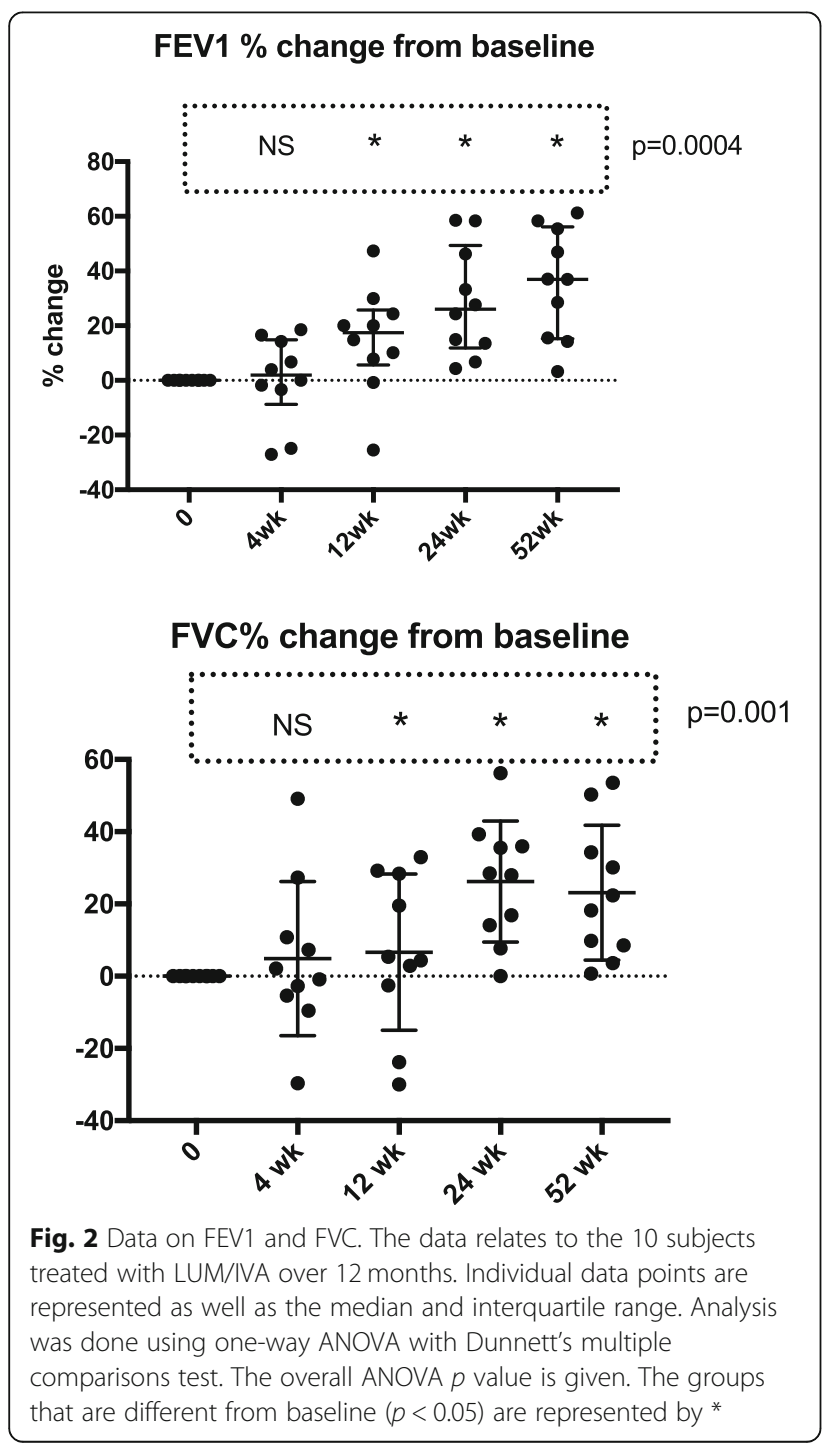

abnormal at baseline, mean 19.7 (SD 7.6). However, there was no significant difference seen in any of these parameters throughout the 12 months. Similarly, the DLCO was measured at the same time points, with no change seen (data not shown).

Adverse events following commencement of LUM/ IVA were reported by $6 / 10$ (60\%). This included symptoms of chest tightness of dyspnoea, that was reported by all 6 within the first $24 \mathrm{~h}$ of commencing treatment. The symptoms persisted intermittently in $3 / 10$ (30\%). Headache was reported to be present intermittently in $2 / 10(20 \%)$. In no cases were symptoms severe enough to warrant discontinuation.

It is our centre's usual practice to assess patients annually when they are clinically stable, with full lung function tests and in those with an FEV $1<50 \%$ predicted, a 6MWT. We therefore had data from a similar cohort, homozygous for Phe508del, with severely impaired airflow obstruction that could be compared over a 12 month period and we used as matched historical controls to the group who had received LUM/IVA (Fig. 4). Exercise capacity was improved at 12 months in those receiving treatment. The 6MWT increased with LUM/ IVA $118 \mathrm{~m}$ (SD 80.9) but decreased in the controls by $61.3 \mathrm{~m}$ (SD 31.1). In the case of the controls the decrease in 6MWT may represent disease progression in those with severe airways disease. For FEV1; LUM/IVA led to an increase of $0.398 \mathrm{~L} / \mathrm{min}$, compared to a fall in the controls - 0.18 (SD 0.2). For FVC; an increase of 0.492 (SD 0.6) compared to a fall of - 0.24 L (0.2). Residual volume fell in those with IVA/LUM, $-0.8 \mathrm{~L}$ (SD 1.1), but rose in the controls by $0.55 \mathrm{~L}$ (SD 0.25). There was no difference in TLC or DLCO. The changes in lung function over 12 months were also associated with changes seen in the 6MWT distance; FEV1 $\mathrm{r}=0.58(p=0.007)$, FVC $\mathrm{r}=0.45$ and RV $\mathrm{r}=-0.64(p=0.02)$ (all, Pearson's correlation coefficient). Finally, we compared exacerbations that had required treatment with intravenous antibiotics. Those treated with IVA/LUM had a mean number of 1.4 (SD 1.1) episodes in the 12 months, compared to 3.3 (SD 1.3) in the controls $(p=0.003)$.

\section{Discussion}

The addition of LUM/IVA to the treatment regime of subjects homozygous for Phe508del with severe airflow obstruction led to a significant improvement in 6MWT distance after 4 weeks, which peaked at 24 weeks and was maintained at 52 weeks. Consistent with the effect seen in subjects with lesser degrees of airflow obstruction there was a small increase, both relative and absolute, in FEV1 and FVC seen by 24 weeks which was maintained at 52 weeks. There were no changes apparent in nitrogen MBW indices, this test was difficult to perform and insensitive to change in these subjects with 
LCI 2.5 over 12 months

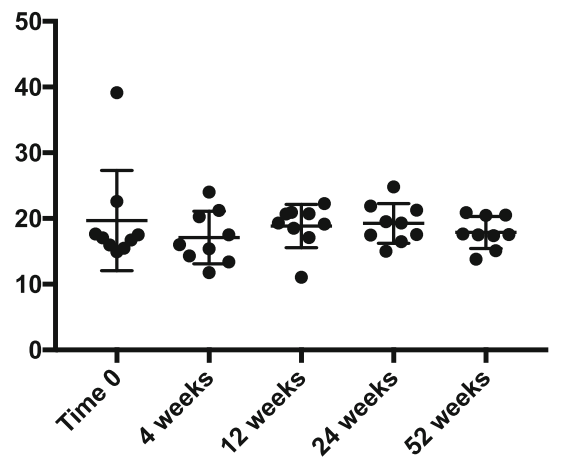

sCOND at Tidal volume

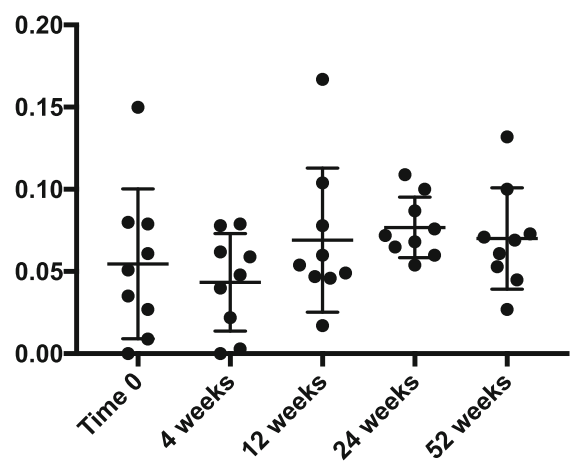

FRC by Multibreath washout

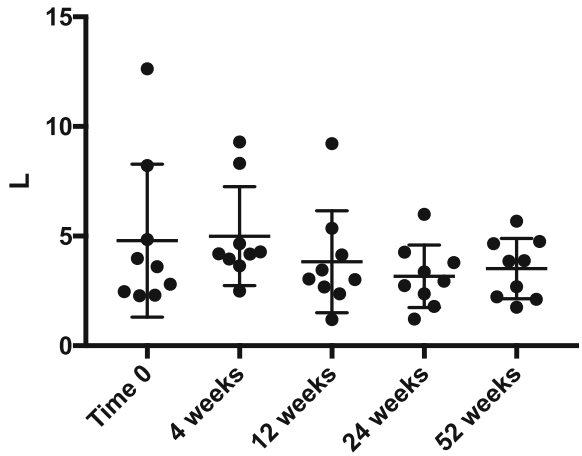

SACIN at Tidal volume

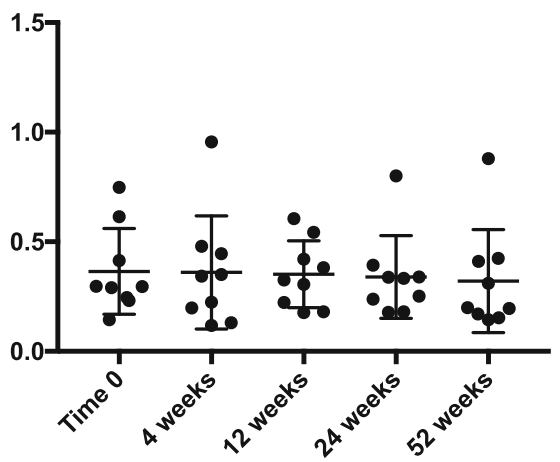

Fig. 3 Data on the Nitrogen MBW. The data relates to the 10 subjects treated with LUM/IVA over 12 months. Individual data points are represented as well as the median and interquartile range. Analysis was done using one-way ANOVA with Dunnett's multiple comparisons test. The overall ANOVA $p$ value is given. No values are given for the $y$ axis as these changes are without dimension

severe lung disease. When compared to historical control subjects over 12 months LUM/IVA resulted in; improved exercise tolerance, improved FEV1, FVC and less air trapping as demonstrated by a fall in RV. The historical controls showed a small but significant deterioration in all these measures over an equivalent 12-month observation period.

While neither spirometry, nor MBW showed detectable change in the initial 4 weeks of LUM/IVA treatment, there was a significant improvement in exercise tolerance; an increase in 6MWT distance (mean increase $=74 \mathrm{~m}$ ), along with improvements in resting heart rate and saturations after 6 min of walking. This effect of treatment was optimal by 6 months and maintained at 12 months (mean increase $=118 \mathrm{~m}$ ). The improvement in 6MWT also correlated with the changes that were seen in lung function after 12 months observation. The improvement seen in 6MWT is clinically meaningful in this cohort with severe lung disease. An increase of between 14 and $30.5 \mathrm{~m}$ in the 6MWT has been determined to be the minimal clinically important distance across a wide range of cardiopulmonary disorders [10]. The value of $6 \mathrm{MWT}$ in CF though is mixed. In patients with mild to moderate airflow obstruction, the $6 \mathrm{MWT}$ distance was found not to be different from that of aged matched healthy controls and tests of maximal exercise capacity are recommended to detect the presence of early disease [11, 12]. However, in more severe CF disease, such as our population, the $6 \mathrm{MWT}$ has been used as a prognostic indicator for transplantation. For example, in 286 consecutive CF patients observed in a retrospective study, the 6MWT distance was shown to reliably correlate with FEV1 and a $6 \mathrm{MWT}$ distance of $<475 \mathrm{~m}$ with desaturation was found to be an independent predictor of death or need for transplant in patients with an ppFEV1 $<60 \%$ [13]. In addition, we are confident the changes that we saw represented a clinically important improvement, they were twice the minimal clinically important difference, sustained to 12 months and correlated closely with the improvement seen in lung function in particular air trapping after 12 months. 
Change in FEV1 at 12 months

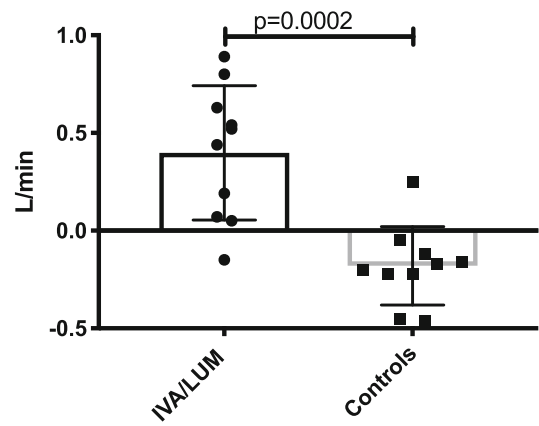

Change in TLC at 12 months

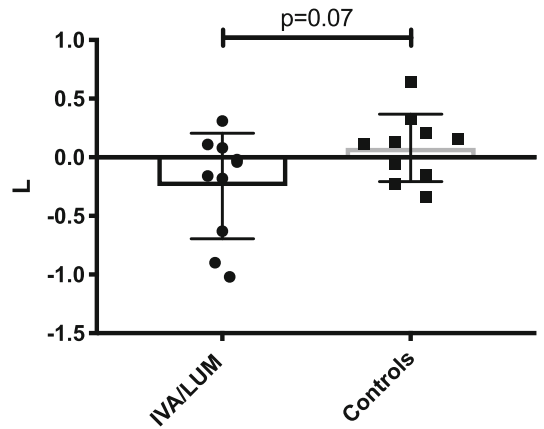

Change in 6MWT after 12 months

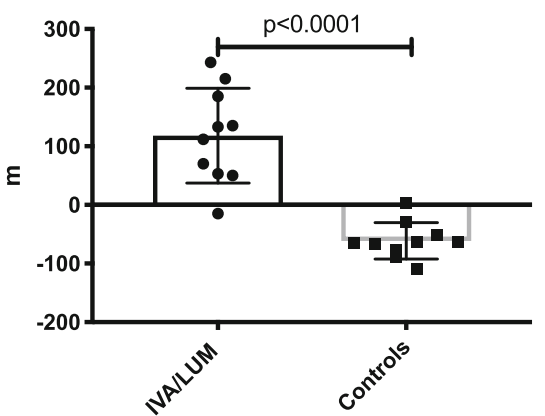

Change in FVC at 12 months

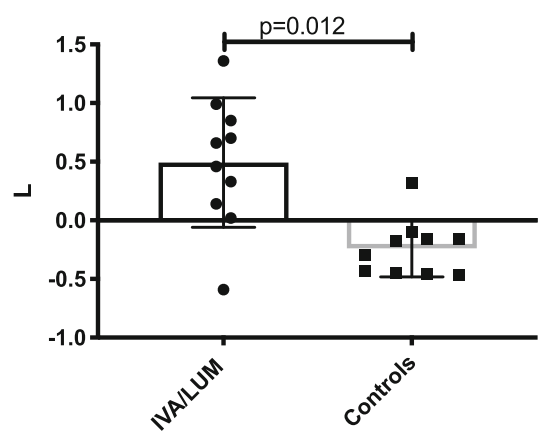

Change in RV at 12 months

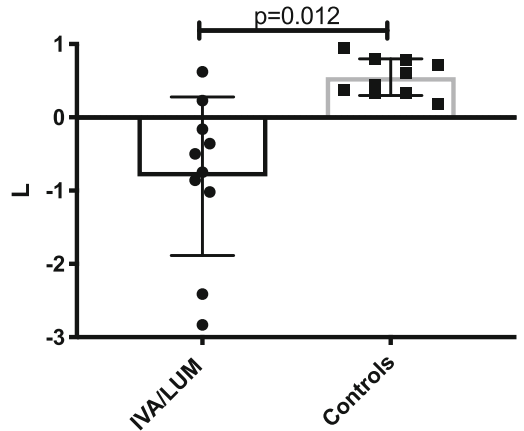

Change in 6MWT baseline HR after 12 months

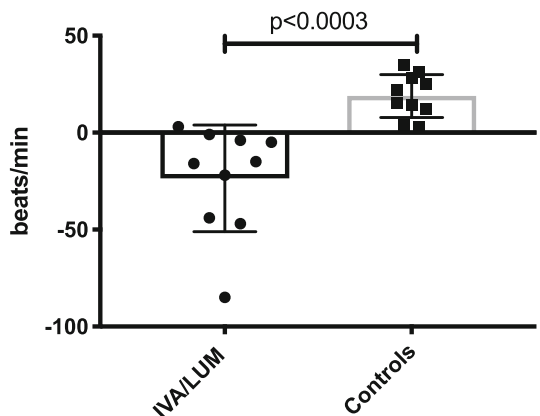

Fig. 4 Comparison in the change seen over 12 months of treatment in 10 subjects treated with LUM/IVA. This is compared to the change seen in 10 age matched historical controls over a 12 month period of review. Data is represented as mean and standard deviation. Comparison made using Student's T test

In comparison the magnitude of the change in lung function seen in the subjects with severe airflow obstruction was small in absolute terms and more variable, but in keeping with previous reports [3,5]. A clear benefit of LUM/IVA did not become apparent until our subjects with severe lung disease completed 6 months of treatment; whereas it has been shown that in subjects with less severe disease (i.e. > 40\% FEV1), to effect lung function within 14 days [3]. With only 10 subjects we were underpowered to determine a difference in lung function in light of the small change in FEV1 that was anticipated with LUM/IVA and the enhanced variability that could be expected with severe lung disease. Despite this with repeated measures and presumably a sustained effect on clinical outcomes, even with this relatively small sample we could see improved lung function by 24 weeks which was sustained at 52 weeks. In CF, airflow obstruction is an important marker of disease severity. A study of risk factors for death of patients with CF suggested that an FEV1 $\leq 30 \%$ predicted, hypercapnoea and the need for enteral nutritional supplements were all associated with a higher risk of death [14]. More recently a review of 
3340 patients of the US CF data registry, showed that adults with an $\mathrm{FEV} 1<30 \%$ predicted had a median survival of 6 years, with the risk of death increased if there was: a need to use oxygen, colonisation with $B$. cenocepacia, a $\mathrm{BMI}<18$, female sex, CF-related diabetes and 1 or more exacerbations in the previous year [15]. Participants in the study described herein did not have airflow obstruction $\leq 30 \%$, were generally well nourished, but did have frequent exacerbations and half of the cohort had diabetes. After 1 year of LUM/IVA treatment none of the subjects had an FEV1 < 30\%. In comparison 30\% (3/ 10) of the control group, had an FEV1 that had decreased to $<30 \%$ after 12 months. By 12 months we could see that this improvement in FEV1 was associated with less air trapping and there also appeared to be fewer serious exacerbations requiring treatment with intravenous antibiotics, compared to the historical controls.

We speculated that in patients with severe airflow obstruction, spirometry change may not be sufficiently sensitive to demonstrate a measurable response to treatment. We therefore utilised nitrogen MBW to see if this technique would allow the earlier detection of changes in lung function, in particular ventilation inhomogeneity, with LUM/IVA. In MBW the derived parameter LCI2.5 (Lung clearance Index 2.5\%) has been used as an index of ventilation inhomogeneity and has been shown to predict early lung disease in children and to correlate, at least in part, to the extent of change present on chest CT [16]. LCI2.5 is a unitless, derivative value calculated as the number of lung volume turnovers required to clear the lungs of the inert marker gas (i.e. nitrogen) to $1 / 40$ th of the starting concentration. We showed that in all cases of LUM/IVA subjects the LCI2.5 was substantially increased compared to predicted values and that there was no consistent improvement evident with LUM/IVA treatment. The MBW has also been used to detect the presence of small airways disease in asthma. Two indices of ventilation heterogeneity in peripheral airways are generated by the technique: one representing the inhomogeneity of ventilation in airways where gas transport is convection-dependent (sCOND) and the other the inhomogeneity of ventilation in more peripheral airways where gas movement becomes diffusion-dependent (sACIN). These measures have been associated in asthma with airway reactivity and neutrophilic inflammation [17]. Again however, LUM/IVA subjects did not demonstrate any improvement in either index. These data may suggest that in our LUM/IVA patients, all of whom had evidence of very severe airflow obstruction and extensive bronchiectasis, the ventilation inhomogeneity was too great and any sensitivity the MBW parameters may offer in the LUM/ IVA-treated lung were lost with the noise and variability seen in this context. This suggestion is supported by the observation that abnormalities in first generation airways can interfere with Scond making anatomical differentiation imprecise. Further, the number of bronchial segments affected by bronchiectasis and LCI has been shown to have a dependent relationship that interferes with LCI $[18,19]$.

Adverse events have been reported with LUM/IVA, and Elborn et al. [5] reported more symptoms of dyspnoea and cough in patients having greater airflow obstruction i.e. ppFEV1 $<40 \%$. Reports have also emerged of an initial fall in lung function in patients with ppFEV $1<40 \%$ receiving LUM/IVA with up to $83 \%$ reporting symptoms of dyspnoea and/or chest tightness [20]. Poor tolerance of LUM/IVA would further limit any small clinical benefit that could be extrapolated from earlier studies to patients with more severe disease. We found in our group of patients that similar respiratoryrelated symptoms were common, though there were no serious adverse events and all participants were able to continue treatment. Given that such events occur more often with LUM/IVA in patients with more severe disease, caution should be exercised during the administration of LUM/IVA in this group of patients. However, if LUM/IVA treatment is tolerated the treatment is beneficial.

There are a number of limitations to our study. Our intervention group was observational and unblinded, as were investigators. They were also not compared to a prospective control arm and participants were not randomised to treatment. However, given the benefits now demonstrated by LUM/IVA in people homozygous with Phe508del with severe lung disease, and where treatment is already optimised such a study would probably be considered unethical and no reliable outcome measure is available in this group to predict response to treatment. However, our historical control group was selected from patients with similar disease severity, had received other similar long-term treatments and apart from treatment with LUM/IVA, were representative of patients with severe lung disease receiving optimal clinical management. Furthermore, the improvements seen in exercise tolerance were sustained and correlated with the small but significant improvement seen in lung function. For these reasons, the comparisons used here appears valid.

\section{Conclusions}

In summary, we have demonstrated that in adults homozygous for Phe508del and with severe lung disease, treatment with LUM/IVA results in early and sustained improvements in the 6MWT and this is indicative of important clinically benefits. Improvement in our cohort was seen in spirometry and as anticipated these changes were small and variable and similar to that shown in the phase III clinical study [3]. 
Importantly, the 6MWT data shown herein suggests that this simple test provides a sensitive and early indicator of the functional impact of LUM/IVA treatment in $\mathrm{CF}$ patients with severe lung disease. It should therefore be considered more often in $\mathrm{CF}$ as an outcome measure in those with severe lung disease for both intervention trials, data registries and regulatory authorities to assess response to treatments.

\section{Abbreviations}

6MWT: Six minute walk test; CF: Cystic Fibrosis; CFTR: Cystic Fibrosis Transmembrane Regulator; DLCO: Single breath carbon monoxide diffusion test; LUM/IVA: Lumcaftor/Ivacaftor; MBW: Nitrogen multiple breath washout

\section{Acknowledgements}

We thank the patients who took part in the assessments, especially in the extra time taken to perform the MBW test.

We thank Vertex for supplying the medication to patients through a compassionate access programme. However, they did not fund the investigators and played no role in devising the investigations or writing the manuscript.

\section{Authors' contributions}

PW devised the study, analysed the data and prepared the manuscript. KC assessed the subjects and performed measures and reviewed the manuscript. TT assessed the subjects and performed measures, collated data and reviewed the manuscript. JB provided advise on MBW, supervised the performance of lung function testing and reviewed the manuscript. DD assessed the subjects and performed measures, collated data and reviewed the manuscript. All authors read and approved the final manuscript.

\section{Funding}

There was no funding provided for this trial.

\section{Availability of data and materials}

The data that support the findings of this study are available from Prof Peter Wark but restrictions apply to the availability of these data, that contain personal information and individuals may be identified. Deidentified data are however is available from the authors upon reasonable request and with permission of Hunter New England LHD Ethics committee.

\section{Ethics approval and consent to participate}

The study was approved by the Hunter New England LHD ethics committee. All participants provided written consent for their information to be used and to deidentified data to be published. Subjects agreed to participate with written informed consent.

\section{Competing interests}

Vertex supplied the medication to patients through a compassionate access programme. However, they did not fund the investigators and played no role in devising the investigations or writing the manuscript.

The authors; PW, KC, JB and TT are employees of Hunter New England LHD. PW is an associate editor for the journal BMC Pulmonary Medicine.

There are no other competing interests to declare.

Received: 14 July 2018 Accepted: 29 May 2019

Published online: 17 June 2019

\section{References}

1. Hull J. Cystic fibrosis transmembrane conductance regulator dysfunction and its treatment. J R Soc Med. 2012;105(Suppl 2):S2-8

2. Van Goor F, Hadida S, Grootenhuis PD, Burton B, Stack JH, Straley KS, et al. Correction of the F508del-CFTR protein processing defect in vitro by the investigational drug VX-809. Proc Natl Acad Sci U S A. 2011;108(46):18843-8.

3. Wainwright CE, Elborn JS, Ramsey BW. Lumacaftor-Ivacaftor in patients with cystic fibrosis homozygous for Phe508del CFTR. N Engl J Med. 2015;373(18):1783-4.

4. McKone EF, Borowitz D, Drevinek P, Griese M, Konstan MW, Wainwright C, et al. Long-term safety and efficacy of ivacaftor in patients with cystic fibrosis who have the Gly551Asp-CFTR mutation: a phase 3, open-label extension study (PERSIST). Lancet Respir Med. 2014;2(11):902-10.

5. Elborn JS, Ramsey BW, Boyle MP, Konstan MW, Huang X, Marigowda G, et al. Efficacy and safety of lumacaftor/ivacaftor combination therapy in patients with cystic fibrosis homozygous for Phe508del CFTR by pulmonary function subgroup: a pooled analysis. Lancet Respir Med. 2016:4(8):617-26.

6. Miller MR, Hankinson J, Brusasco V, Burgos F, Casaburi R, Coates A, et al. Standardisation of spirometry. Eur Respir J. 2005;26(2):319-38.

7. Graham BL, Brusasco V, Burgos F, Cooper BG, Jensen R, Kendrick A, et al. 2017 ERS/ATS standards for single-breath carbon monoxide uptake in the lung. Eur Respir J. 2017;49(1):1600016. https://doi.org/10.1183/13993003. 00016-2016

8. Wanger J, Clausen JL, Coates A, Pedersen OF, Brusasco V, Burgos F, et al. Standardisation of the measurement of lung volumes. Eur Respir J. 2005:26(3):511-22.

9. Holland AE, Spruit MA, Troosters T, Puhan MA, Pepin V, Saey D, et al. An official European Respiratory Society/American Thoracic Society technical standard: field walking tests in chronic respiratory disease. Eur Respir J. 2014;44(6):1428-46.

10. Bohannon RW, Crouch R. Minimal clinically important difference for change in 6-minute walk test distance of adults with pathology: a systematic review. J Eval Clin Pract. 2017:23(2):377-81.

11. Ziegler B, Rovedder PM, Oliveira CL, de Abreu e Silva F, de Tarso Roth Dalcin P. Repeatability of the 6-minute walk test in adolescents and adults with cystic fibrosis. Respir Care. 2010;55(8):1020-5.

12. Vandekerckhove K, Keyzer M, Cornette J, Coomans I, Pyl F, De Baets F, et al. Exercise performance and quality of life in children with cystic fibrosis and mildly impaired lung function: relation with antibiotic treatments and hospitalization. Eur J Pediatr. 2017;176(12):1689-96.

13. Martin C, Chapron J, Hubert D, Kanaan R, Honore I, Paillasseur JL, et al. Prognostic value of six minute walk test in cystic fibrosis adults. Respir Med. 2013;107(12):1881-7

14. Belkin RA, Henig NR, Singer LG, Chaparro C, Rubenstein RC, Xie SX, et al. Risk factors for death of patients with cystic fibrosis awaiting lung transplantation. Am J Respir Crit Care Med. 2006;173(6):659-66.

15. Ramos KJ, Quon BS, Heltshe SL, Mayer-Hamblett N, Lease ED, Aitken ML, et al. Heterogeneity in survival in adult patients with cystic fibrosis with FEV1 $<30 \%$ of predicted in the United States. Chest. 2017;151(6):1320-8.

16. Subbarao P, Milla C, Aurora P, Davies JC, Davis SD, Hall GL, et al. Multiplebreath washout as a lung function test in cystic fibrosis. A Cystic Fibrosis Foundation workshop report. Ann Am Thorac Soc. 2015;12(6):932-9.

17. Farah CS, Keulers LA, Hardaker KM, Peters MJ, Berend N, Postma DS, et al. Association between peripheral airway function and neutrophilic inflammation in asthma. Respirology. 2015;20(6):975-81.

18. McNulty W, Usmani OS. Techniques of assessing small airways dysfunction. Eur Clin Respir J. 2014;1.

19. Verbanck S, King GG, Zhou W, et al. The quantitative link of lung clearance index to bronchial segments affected by bronchiectasis. Thorax. 2018;73:82-4

20. Popowicz N, Wood J, Tai A, Morey S, Mulrennan S. Immediate effects of lumacaftor/ivacaftor administration on lung function in patients with severe cystic fibrosis lung disease. J Cyst Fibros. 2017;16(3):392-4.

\section{Publisher's Note}

Springer Nature remains neutral with regard to jurisdictional claims in published maps and institutional affiliations.

Ready to submit your research? Choose BMC and benefit from:

- fast, convenient online submission

- thorough peer review by experienced researchers in your field

- rapid publication on acceptance

- support for research data, including large and complex data types

- gold Open Access which fosters wider collaboration and increased citations

- maximum visibility for your research: over $100 \mathrm{M}$ website views per year

At $\mathrm{BMC}$, research is always in progress.

Learn more biomedcentral.com/submissions 